\title{
INVESTIGACIONES
}

\section{Discursividades binarias en las políticas educativas de género y sexualidad en Chile*}

\author{
Binary discursivities in educational policies on gender \\ and sexuality in Chile
}

\author{
Karen Mardones Leiva ${ }^{a}$, Marcela Apablaza Santis ${ }^{b}$, Pamela Vaccari Jiménez ${ }^{c}$ \\ ${ }^{a}$ Candidata a Doctora en Ciencias Humanas, Facultad de Filosofía y Humanidades \\ y Profesora del Instituto de Estudios Psicológicos, Facultad de Medicina, Universidad Austral de Chile. \\ karen.mardones@uach.cl \\ ${ }^{b}$ Doctora en Ciencias de la Educación. Académica del Instituto de Aparato Locomotor y Rehabilitación, \\ Facultad de Medicina, Universidad Austral de Chile. \\ marcela.apablaza@uach.cl
}

${ }^{c}$ Doctora en Psicología Social. Profesora del Departamento de Psicología, Facultad de Ciencias Sociales, Universidad de Concepción. pamelavaccari@udec.cl

\begin{abstract}
RESUMEN
Las políticas producen y reproducen determinados sistemas de valores que reflejan ciertas definiciones de realidad; por esto son actividades discursivas que contribuyen a sedimentar/objetivar/institucionalizar algunas expresiones como lo correcto, lo obvio, lo normal. Asumimos una posición cuestionadora de esta normalización, buscando politizar órdenes de género y sexualidad atados a binarismos sexogenéricos. Analizamos -desde una perspectiva posestructuralista- los discursos de las políticas educativas de género y sexualidad en Chile durante la presente década, indagando en cuatro documentos elaborados por el Ministerio de Educación de Chile. El análisis devela la existencia de normatividades expresadas en un dimorfismo sexual ideal anclado en la biología, perpetuando el binarismo hombre/mujer-niño/niña, y la heterosexualidad como la vía aceptada y valorada de expresión del deseo. Apostamos por potenciar la reflexión y deconstrucción de las formaciones discursivas sobre los cuerpos sexuados, problematizando las normas construidas y sostenidas, y eliminar los binarismos que persisten en las discursividades textuales revisadas.
\end{abstract}

Palabras claves: dimorfismo sexual, normalización, posestructuralismo, deconstrucción.

\begin{abstract}
Policies produce and reproduce certain value systems which reflect certain definitions of reality; they are therefore discursive activities that help to seat/objectivise/institutionalise some expressions as correct, obvious or normal. We question this normalization, seeking to politicise orders of gender and sexuality tied to sex-gender binarisms. We analyse, from a post-structuralist perspective, the discourses of educational policies on gender and sexuality in Chile during the present decade, studying four documents produced by the Chilean Education Ministry. Our analysis reveals the existence of normativities expressed in an ideal sexual dimorphism anchored in biology,

\footnotetext{
* Agradecimientos a por la Agencia Nacional de Investigación y Desarrollo / Programa Becas / Doctorado Nacional 2017 folio 21170553. Proyecto Anillos de Investigación en Ciencias Sociales y Humanidades (SOC180045) "Horizontes convergentes: producción, mediatización, recepción y efectos de las representaciones culturales de la marginalidad”. Además, este artículo contó con el apoyo de la Agencia Nacional de Investigación y Desarrollo, a través del Proyecto FONDECYT 11170479, "Prácticas 'psi' en la escuela: efectos de la intervención psicosocial en el marco de políticas educativas inclusivas".
} 
perpetuating the man/woman-boy/girl binarism and heterosexuality as the accepted and respected path for the expression of desire. Our object is to encourage reflection on and deconstruction of discursive formations on sexed bodies, problematising the norms constructed in the past and still sustained; and to eliminate the binarisms that persist in the texts reviewed.

Key words: sexual dimorphism, normalization, poststructuralism, deconstruction.

\section{LAS POLÍTICAS COMO ACTIVIDADES DISCURSIVAS}

En un marco internacional que promulga el Enfoque de Derechos Humanos para abordar diferentes problemáticas a nivel internacional, el Estado de Chile ha asumido desde los años noventa una agenda política que lo ha situado dentro del grupo de países que avanzan hacia la igualdad y la justicia social. Para expresar su compromiso con dichas metas, Chile se ha adherido a una serie de convenciones internacionales. Las diversas ratificaciones han conllevado la implementación de políticas, planes y programas en diferentes áreas del desarrollo social del país.

Es en este contexto que las políticas educativas de inclusión se suman a las importantes reformas que se realizan con el objetivo de adecuarse a los estándares internacionales de desarrollo. Con respecto a la temática de género y educación, el Ministerio de Educación del Gobierno de Chile [MINEDUC] inscribe sus políticas en un marco normativo nacional e internacional, que le da el soporte legal para la elaboración de las políticas en ese ámbito. Para responder a dichos marcos, el MINEDUC creó en el año 2014 la Unidad de Equidad de Género [UEG]:

Como una estructura permanente y transversal encargada de impulsar la incorporación de la perspectiva de género en las políticas, planes y programas ministeriales, con el fin de avanzar en una educación pública de calidad e inclusiva, que permita el desarrollo integral y equitativo de niños, niñas y jóvenes del país (2015, p. 7).

Desde la perspectiva posestructuralista se entiende que las políticas actúan como 'prácticas discursivas' (Bacchi, 2007, p. 47 en Infante, Matus y Vizcarra, 2011). Por esto no son procedimientos neutros ni apolíticos, sino redes discursivas en cuya materialidad se definen individuos, comportamientos y posibilidades de participación (Allan, 2003 en Infante et al. 2011). En palabras de Infante et al. (2011) "Las políticas están orientadas por ciertos valores y representan supuestos específicos en relación con el objeto de su promulgación" (p. 6). Es por esto por lo que las políticas producen y reproducen determinados sistemas de verdad que con el tiempo se transforman en definiciones de realidad.

Bajo este marco, el discurso lo entendemos "como prácticas o sistemas de poder/ conocimiento que son creados histórica y culturalmente localizados" (Infante et al. 2011, p. 146). El interés por el discurso es transversal a diferentes disciplinas y lo es, del mismo modo, en los debates de las ciencias humanas contemporáneas en donde la centralidad del lenguaje y del discurso son crecientes (Iñiguez, 2005). Lo anterior da cuenta de la existencia de un campo de lucha de éstos por el significado, en tanto cada vez más se visibilizan prácticas discursivas que reclaman ser escuchadas y que sus significados circulen del mismo modo en los diversos espacios sociales. 
Michel Foucault y Judith Butler son figuras influyentes del movimiento intelectual denominado posestructuralismo, caracterizado entre otras ideas por la crítica a las jerarquías binarias, al lugar decisivo de los discursos vinculados a las relaciones de poder que cristalizan en la construcción de la realidad social, y la relevancia de la historicidad en ese proceso. Con esto ofrecen interesantes perspectivas para el análisis social, invitándonos a cuestionar y a denunciar los diversos mecanismos de opresión que se han instalado, consagrado y naturalizado en el orden de la cultura. Si tomamos como referente la tesis principal de Lyotard (2000) sobre la condición posmoderna, tanto en Butler como en Foucault es patente la desconfianza hacia los metarrelatos, los que cabe entender como supranarrativas o metadiscursos que, para Lyotard (2000) -y en consecuencia para Foucault y Butler- no son más que fábulas susceptibles de ser desmanteladas.

Foucault, en El orden del discurso (2010), visibiliza que todo discurso tiene un contexto de producción, que hay ciertas condiciones de posibilidad para que este no sólo se distribuya, sino también para que se produzca, es decir, para que siga un orden ya definido. Con este planteamiento problematiza sobre cualquier tema que ha sido entendido como lo natural, establecido y correcto, develando que hay condiciones que impiden la emergencia y circulación de discursos que rompan con dicho orden. Es por esto lo que "uno sabe que no tiene derecho a decirlo todo, que no se puede hablar de todo en cualquier circunstancia, que cualquiera, en fin, no puede hablar de cualquier cosa" (Foucault, 2010, p. 14). A la vez nos señala que los sistemas de exclusión de discursos, aquellos prohibidos en una sociedad, se apoyan en una base institucional, incluso científica y disciplinaria (Foucault, 2010).

En La arqueología del saber, el pensador francés alude igualmente a este contexto de producción posibilitador de discurso, al que le llama formación discursiva y la entiende como un:

haz complejo de relaciones que funciona como reglas: prescribe lo que ha debido ponerse en relación, en una práctica discursiva, para que ésta se refiera a tal o cual objeto, para que ponga en juego tal o cual enunciado, para que utilice tal o cual conjunto, para que organice tal o cual estrategia. Definir en su individualidad un sistema de formación es, pues, caracterizar un discurso o un grupo de enunciados por la regularidad de una práctica (Foucault, 2002, p. 122-123).

De esta manera, los sujetos se encuentran constreñidos por las formaciones discursivas sobre la construcción sociocultural de sus cuerpos, formaciones que su sociedad y su tiempo les imponen; respondiendo a reglas del deber ser que actúan como límites que dibujan sus posibilidades de acción y pensamiento, que actúan por lo tanto como mecanismos de control operando en las estructuras cognitivas y de ese modo en las subjetividades. De ese modo, la producción de identidades queda confinada a las posibilidades discursivas (Infante et al., 2011).

Desde ahí es que entendemos el campo educativo como reproductor de ciertos órdenes sociales y culturales (Matus y Haye, 2015). Tomando la invitación de Matus y Haye (2015) en este artículo pretendemos abrirnos al ejercicio intelectual de producir conocimiento acerca de la normatividad sexo/genérica, reproducida a través de las políticas que son pregonadas desde el ministerio y contenidas en los documentos orientadores de las prácticas en escuelas, y en este caso particular, de aquellas vinculadas al género y a la sexualidad. 


\section{INDAGACIÓN DISCURSIVA TEXTUAL}

En este apartado realizamos un análisis del discurso de cuatro documentos enmarcados dentro de las políticas educativas de género y sexualidad en Chile durante la presente década, intentando responder a ¿qué definiciones de la realidad contienen?, ¿qué valores y/o supuestos subyacen en sus discursos?, ¿con qué otras definiciones de realidad se conectan?; intentando develar aquello que se encuentra detrás de lo aparente. Específicamente tomamos cuatro documentos elaborados por el MINEDUC que abordan explícitamente conceptos de género y sexualidad: del año 2012 el texto "Enfoque de género en el sistema educativo", del año 2013 el texto "Formación en sexualidad, afectividad y género"; del año 2015 el texto "Educación para la igualdad de género. Plan 2015-2018"; y del año 2017 la circular sobre "Derechos de niñas, niños y jóvenes trans en el ámbito de la educación".

Globalmente, las políticas de género han sido abordadas principalmente bajo una mirada despolitizada (Youdell, 2011), enmascaradas bajo el lenguaje de diversidad (Ahmed, 2011), de equidad (Fraser, 2008) y en respuesta a problemáticas de desigualdad social. En este marco se elaboran políticas públicas bajo tres líneas de acción: las políticas de igualdad de oportunidades, de acción afirmativa y de transversalidad y paridad (Arriagada, 2006). Éstas apuntan, desde distintos énfasis, a mejorar las condiciones de participación de las mujeres, a minimizar y a revertir la discriminación y a generar acciones transversales como Estado nación.

Específicamente en Chile, las políticas de género se han restringido históricamente hacia las mujeres y se han abordado de manera secundaria, centradas principalmente en la generación de mejores condiciones para el acceso y aumento de cobertura escolar. Hasta ahora no se ha puesto mayor atención en modificar e intervenir las condiciones desigualitarias de participación y desarrollo entre las mujeres -y sólo recientemente, tras las movilizaciones estudiantiles del 2018- se ha abierto la posibilidad de cuestionar los procesos educativos cimentados en una educación sexista (Matus, 2018).

Si bien han habido avances en los últimos 20 años, la evaluación de su impacto aún es deprimente, pues persisten prácticas pedagógicas sexistas, al alero de un currículum oculto que las sostienen y reafirman, así como ausencia de una política clara y fuerte sobre los asuntos de género (Provoste, 2006; Galaz, Troncoso y Morrison, 2016), dado que las comunidades educativas y las administraciones señalan falta de conocimiento sobre las estrategias de implementación y escasa comprensión sobre el enfoque de género, manteniendo una mirada relativizadora y minimizadora respecto de la importancia y necesidad de implementar acciones sustantivas.

Por consiguiente, en la actualidad, el marco político vigente se focaliza en el área de convivencia escolar, donde género y sexualidad, es una más de las temáticas abordadas. Asimismo, se mantiene como premisa la tríada sexo/género/sexualidad, lo que puede ser desprendido del cuerpo legal vigente y del material de apoyo respectivo ilustrado en los documentos analizados a continuación.

En los cuatro documentos constatamos una crítica al sistema de correspondencia natural -orden obligatorio- del sexo con el género. Dicho orden fue expuesto por la antropóloga feminista Gayle Rubin en los años setenta, al identificar la existencia de un sistema sexo/ género -orden sexogenérico- en toda sociedad, definiéndolo como aquel "sistema de relaciones sociales que transforma la sexualidad biológica en productos de la actividad humana y en el que se satisfacen esas necesidades humanas transformadas" (Rubin, 1986, 
p. 97). Los documentos exponen que una mujer no necesariamente debe ser femenina, así como un hombre tampoco debe ser masculino. En ese sentido se hace la crítica que ya hicieran las feministas del último tercio del siglo XX, sobre la construcción social de la diferencia sexual (Lamas, 2015), enfatizando que nacer con una determinada biología no implica habilidades particulares para ciertos espacios y actividades -para hombres y mujeres- por naturaleza; sino que dicha división responde a una construcción sociocultural e histórica. El documento del año 2017 avanza aún más al referirse a estudiantes "trans", señalando que son "personas cuya identidad y/o expresión de género no se corresponde con las normas y expectativas sociales tradicionalmente asociadas con el sexo asignado al nacer" (p. 2). Introduce con ello una discusión que va más allá de lo que los anteriores documentos contenían. En este caso la identidad de género, vale decir la vivencia interna de género, puede no corresponderse a lo socialmente esperado de acuerdo con el sexo asignado por la medicina. Con esto ser hombre o mujer no está dada por el sexo biológico, sino por el sentido de pertenencia uno en particular.

En segundo lugar, los documentos analizados aluden, especialmente en MINEDUC (2012), a las brechas entre hombres y mujeres, expresadas en cifras de acceso a espacios educativos (ya sea para estudiar o trabajar) y a análisis comparativos de su rendimiento en diferentes actividades y espacios. La información es entregada como una denuncia de algo que no debiese ocurrir en el sistema educativo chileno, e invitan a reconocer que hombres y mujeres tienen las mismas capacidades, el mismo potencial para aprender y por lo tanto deben tener las mismas oportunidades para acceder a la educación, así como a espacios laborales. Por ejemplo, en MINEDUC (2013) se indica que "la igualdad de oportunidades entre hombres y mujeres es importante porque se reconoce y se brinda tanto a hombres como a mujeres los mismos espacios, posibilidades y recursos para que desarrollen en plenitud todo su potencial" (p. 22). Como sostiene Apablaza (2015), asistimos hoy a un mayor cuestionamiento de estereotipos de género existentes en la escuela y a la desigualdad de oportunidades que ello provoca, sin embargo, hay discursos esencialistas y naturalizados que no son puestos en el debate. A esto nos referimos en las siguientes líneas.

Si bien los dos puntos indicados hasta aquí son leídos por gran parte de los movimientos de mujeres y los movimientos feministas como importantes avances, resultan ser controversiales si los analizamos desde las discusiones y demandas actuales de los estudios de género, especialmente desde la teoría queer. Esta teoría desarma preceptos que la teoría de género había promovido, dando un giro radical a las categorías convencionales de sexualidad y género (Ambrosy, 2012). Ello porque éstos se develan como instrumentos que norman la sexualidad humana, expresados en un dimorfismo sexual ideal. Por ejemplo, en MINEDUC $(2012,2013)$ se define al sexo como "el conjunto de características biológicas que nos define como mujer u hombre" (p. 28), objetivando una norma dicotómica y de ese modo perpetuando un binarismo sexual. Conjuntamente, en todos los documentos se encuentran discursos reiterados aludiendo a "niños y niñas", así como a "mujeres y hombres". Con ellos se refuerza una estructura de pensamiento rígida -el binomio hombre/ mujer y niño/niña- que se posiciona como lo 'normal', aquello que no se cuestiona, es decir no aparece problematizada la segmentación entre esas -sólo- dos opciones posibles. Inclusive en la circular del año 2017, en muy pocas ocasiones se utiliza algún término genérico/inclusivo como "personas", pues en la mayor parte de las ocasiones se alude a la habitual y naturalizada dicotomía "niños y niñas", donde lo trans pasa a ser lo diferente respecto del binomio normalizado. 
En ese sentido, como sostienen Matus e Infante (2011) al insistir en diferencias entre grupos no se problematiza la noción de 'normalidad' frente a la cual se construyen estas diferencias. De ese modo la noción de 'normal' es naturalizada, debiendo las demás identidades -las otras- asirse a ella. Es por esto por lo que Foucault (2000) nos refiere que la norma "trae aparejados a la vez un principio de calificación y un principio de corrección. Su función no es excluir, rechazar. Al contrario, siempre está ligada a una técnica positiva de intervención y transformación, una especie de proyecto normativo" (p. 57). Esa diferencia, esa otredad, se observa, se diagnostica -se marca-, se tolera e incluso se pretende 'mejorar' y con ello se les inscribe en su otredad de desviados, marginados, indeseables, en definitiva, inferiores (Matus y Haye, 2015). Lo diferente es lo desviado respecto de la norma, ese Otro sigue siendo lo anormal, el des-vío, quien se aparta de la vía, de la vía principal (Ramírez Pavelic y Contreras Salinas, 2016). Apablaza (2015) señala que la inscripción de estos discursos de normalidad en las políticas educativas, van definiendo los modos de relación aceptables y la configuración de sujetos constreñidos a aquellos. Por esto Ramírez Pavelic y Contreras Salinas (2016) sostienen que no cuestionar la misma norma, conduce a que en los contextos educativos la diferencia sea entendida como indisciplina, puesto que "la diferencia viene a ser experimentada más bien como un distanciamiento de la norma" (p. 245) y por tanto los sujetos LGTB experimenten la diferencia como no ajustarse a la heteronormatividad impuesta en el espacio educativo. De esta forma las autoras citadas señalan que es justamente este espacio el de mayor complejidad para asumir la condición LGTB entre el estudiantado.

Por otro lado, otro aspecto para problematizar, en MINEDUC (2013) -al referirse a los roles y estereotipos sexuales-, se indica: "el rol del hombre y de la mujer en la sociedad, el valor de la complementariedad entre los sexos, igualdad de oportunidades, derechos y deberes, etc." (p. 11). Además de reiterar el binomio antes aludido: hombre/mujer, nos señala "el valor de la complementariedad", dejando entrever una implícita división entre hombres y mujeres, aludiendo a que cada cual hace una parte que complementa a la otra; entonces nos preguntamos a qué división se refieren: ¿espacios?, ¿actividades? Así, hombres y mujeres son llamados a alcanzar la valorada complementariedad de los roles. La peligrosa alusión a la complementariedad implica la perpetuación de la segmentación horizontal y vertical en los variados espacios laborales, educativos e incluso domésticos. El binarismo sexual como norma conecta con otros binarismos expuestos dentro de los estudios de género: hombres/mujeres sería a público/privado, cultura/naturaleza o productivo/reproductivo, respectivamente. Asimismo, la trampa de la complementariedad refuerza la norma heterosexual, la norma reproductiva en las relaciones erótico-afectivas.

Otro punto de análisis en los documentos indagados son las alusiones a la pluralidad de manifestaciones de la sexualidad humana. En los documentos de 2012 y 2013 no se encuentra señalada esta perspectiva, como sí es posible observar en el documento del año 2015 y en el del 2017. En MINEDUC (2015) se indica que la UEG "busca resguardar la igualdad en el ejercicio de sus derechos; la construcción de espacios de convivencia libres de discriminación y violencia de género; la inclusión de todas las identidades y expresiones de género y orientaciones sexuales" (p. 7). Asimismo, se expone, que la construcción cultural de la diferencia sexual nos define "....como hombres y mujeres con identidades, expresiones y orientaciones diversas” (p. 9). Más adelante, en la página 11, se especifica respecto al sexo indicando como opciones "hembra, intersexual y macho" y en lo que refiere a la identidad de género se señalan "mujer, transgénero, transexual, hombre 
y cisgénero". Considerando lo anterior, es posible leer que el texto de 2015 imprime una nueva perspectiva con relación a los documentos de los años anteriores, por la referencia a otras manifestaciones sobre los cuerpos e identidades; sin embargo, las alusiones a estas posibilidades se limitan a un par de párrafos. En las restantes páginas el documento no hace referencia a esa multiplicidad de configuraciones, más bien se reiteran las opciones binarias aludidas en párrafos anteriores: "niños y niñas" y "hombres y mujeres". Con ello se devela que la apertura hacia significados plurales es más bien aparente, al cruzarse y tensionarse con discursos contrarios que insisten en el orden normativo dicotómico.

Todos los documentos revisados perpetúan una definición de la realidad binaria para los sexos, más allá de las escasas referencias a las manifestaciones plurales, puesto que aún ellas son la diferencia respecto a la norma. De ese modo el binarismo se consagra como lo normal: se es niño o niña, se es hombre o mujer, se debe serlo. Una de esas dos categorías debe ser adscrita e inscrita en nuestros cuerpos, pues la norma sexual así lo indica.

\section{DISCUTIENDO / PROBLEMATIZANDO LA NORMA}

¿Sólo es posible identificar dos sexos?, ¿debemos identificarnos con uno en particular? La historia -pasada y actual- nos muestra que hay personas que rompen la normatividad del dimorfismo. Foucault (1985) nos presenta en su libro sobre Herculine Barbin la experiencia biográfica de un hermafrodita que vivió a fines del siglo XIX, junto a la insistencia médica y judicial para asignarle un 'sexo verdadero'. Actualmente, llamados intersexuales, son personas no-binarias que desestabilizan la norma sexual dominante. Desplazándose aún más, actualmente se discute y se visibiliza la existencia de un abanico mayor de sexos (Raíces Montero, 2015); de acuerdo con Zúñiga (2017), la variedad de combinaciones entre la carga cromosómica y hormonal, y los genitales externos de un sujeto, no es reflejada por el uso de las categorías hombre o mujer. Durante la fecundación se define un conjunto de cromosomas que pare ser viables tienen que contener un cromosoma $\mathrm{X}$, se pueden dar lugar a combinaciones XX, XY (las más frecuentes) y otros conjuntos, más escasos pero existentes (como XXY o X delección de X). Así, podría decirse que hay cinco sexos: hombre, mujer, herms (nacidos/as con un testículo y un ovario) merms (nacidos con testículos, pero también con indicios de genitales femeninos) y ferms (nacidos con ovarios, pero con algunos aspectos de genitales masculinos). Esto último es lo que se ha dado en denominar intersexualidad. Durante el siglo XX y actualmente, la ciencia biomédica ha planteado discusiones sobre la difusidad del sexo (Martin Casares, 2018). Por esto, desde el punto de vista biomédico, no puede dividirse tan rígidamente como se pretende, no se pueden establecer limitaciones estrictas basadas en las diferencias biológicas. Sin embargo, en el imaginario colectivo el sexo se presenta como dual e inmutable, por lo que las realidades múltiples quedan ocultas bajo la perpetuación del dimorfismo sexual. El velo que las oculta toma múltiples formas, desde la consideración de anomalías médicas que deben ser corregidas quirúrgicamente; pasando por políticas que escasamente las nombran o si lo hacen es para reafirmar que son la otredad periférica; hasta las situaciones de la vida cotidiana en donde se les esconde e invisibiliza.

En la antropología -como disciplina pilar en las discusiones sobre sexo y género, así como en el cuestionamiento contemporáneo sobre la dualidad naturaleza/cultura- llama la atención que se discuta escasamente sobre el sexo como entidad estrictamente biológica 
y prácticamente no se le niegue como tal. Incluso, como sostiene Aurelia Martín Casares (2018) no existe ninguna intención de suprimir del vocabulario antropológico la palabra "sexo", como si lo hiciese con la palabra "raza", tan debatida justamente por su anclaje en la biología y actualmente excluida. De esta manera cabe preguntarse a qué responde la dificultad de deconstruir la palabra sexo, qué sistemas y órdenes desestabiliza y perturba (Martín Casares, 2018). Foucault (2010) nos dirá que la sexualidad es un tema prohibido por excelencia, pues es precisamente allí "donde se multiplican las casillas negras" (p. 15).

Los discursos analizados en este escrito se encuentran dentro de una red más amplia y compleja de significados y prácticas siendo reflejo de las tensiones, luchas y contradicciones que ocurren en nuestra sociedad. Es necesario indicar que Chile es un país que, respecto de los temas vinculados a la sexualidad, aún en la actualidad, presenta posiciones muy conservadoras. Estas posiciones se encuentran ancladas en una impronta religiosa y política que las ampara. Así, temáticas como el divorcio en su momento, el aborto, el matrimonio igualitario y la adopción homoparental, entre otros temas en la actualidad, son importantes espacios y discursos en pugna. Entonces, el cuestionamiento de la norma resulta amenazante y desestabilizador, por lo mismo se evita problematizarla, tensionarla y subvertirla.

Desde una perspectiva que podríamos considerar posestructuralista intermedia (Botero y Duque, 2012), Berger y Luckmann dan a conocer en 1967 su obra La construcción social de la realidad. Allí plantean que la realidad social ha sido construida intersubjetivamente a partir de relaciones sociales que se han objetivado a través del tiempo en procesos como la habituación y la rutinización, llegando a institucionalizarse. Dicho producto se transforma en realidad objetivada, que vuelve a actuar sobre su productor a través de la socialización, durante la que se internalizan esas objetivaciones originadas en las iniciales relaciones compartidas (Berger y Luckmann, 2008). Así, ambos pensadores sostendrán que "las tipificaciones compartidas se construyen en el curso de la historia compartida: no pueden crearse en un instante. Comprender qué es una institución pasa por comprender el proceso histórico por el cual se produjo" (p. 74). Nuestras sociedades han objetivado el binarismo de los sexos, aceptando -en el tiempo- la existencia de sólo dos: hombre y mujer (macho y hembra), institucionalizándose como la norma.

Cabe preguntarse entonces ¿qué es el sexo? Siguiendo a Butler (2017) ¿es el sexo natural, anatómico, cromosómico u hormonal?, “¿existe una historia de cómo se determinó la dualidad del sexo, una genealogía que presente las opciones binarias como una construcción variable?" (p. 55). La dualidad de los sexos se nos ha presentado como prediscursiva, es decir, anterior a la cultura; sin embargo, el sexo también es discursivo -así como el género-, pues los cuerpos han sido interpretados mediante significaciones y con ello no todas las interpretaciones alternativas han tenido la posibilidad de ser consideradas, pues los límites han estado puestos dentro de la estructura binaria (Butler, 2017). Preguntarse, por ejemplo, por lo trans en los contextos educativos, es preguntarse por una realidad no tan rígida y sî desprendida de la norma sexual. Lo mismo por transgresiones al disciplinamiento de las corporalidades, como son los hombres con vagina o las mujeres con pene, posibilidades aquellas que desarman el binomio hombre/mujer anclado a una determinada genitalidad. En palabras de Butler (2017), manifestaciones como estas contribuyen con el propósito de extender la legitimidad a "los cuerpos que han sido vistos como falsos, irreales e ininteligibles" (p. 29). Los cuerpos que rompen con el dimorfismo son propuestas que invitan a nuevas definiciones de la realidad y a preguntarse por qué éstas no han tenido el 
'privilegio' de ser nombradas como la 'normal'. Concretamente en las escuelas no existen los espacios para que los sujetos LGTB se expresen libremente, por ejemplo se les obliga a hacer la fila junto al grupo del sexo que les fue asignado al nacer y no con el grupo con que se identifican, al que se sienten pertenecientes; no poder disfrazarse de princesa si se es hombre; o que se cuestionen, e incluso repudien, las muestras de afecto LGTB, mientras que las manifestaciones heterosexuales son permitidas (Ramírez Pavelic y Contreras Salinas, 2016). De esta forma, Matus e Infante (2011) invitan a problematizar sobre "quiénes" son aquellos que están dictando las normas y sobre quién o quiénes van a ser incluidos y por cuáles diferencias. Señala Foucault (2010) que la mantención del orden establecido se sostiene sobre una red institucional e incluso científica, que asegura y resguarda que ese orden normativo dominante se reproduzca y se mantenga. Precisamente instituciones como el estado, la familia y la escuela, son las encargadas de esto. De ahí la necesidad política de descascarar las discursividades normativas que estas instituciones han ido sedimentando.

Al comenzar el libro sobre Herculine Barbin, Foucault pregunta: “ $¿ T e n e m o s$ necesidad de un sexo verdadero?" (Foucault, 1985, p. 11), justamente para problematizar sobre esa norma sexual que se ha asumido como natural y necesaria, y con ello cuestionar hasta qué punto y para quiénes es importante. En la medida que no se problematice la propia construcción del sexo, es decir, su normalización binaria, las políticas educativas de género y sexualidad continuarán perpetuando la creencia de un sexo verdadero. Por esto resuena con sentido lo que Kumashiro (2002 en Matus e Infante, 2011) denuncia al señalar que:

A pesar de que un currículo que busca inclusión pueda tener éxito en enseñar que el Otro es tan normal o importante que la norma, no necesariamente cambia la propia definición de 'normal' y las maneras en que tradicionalmente nos vemos nosotros mismos como tales. (p. s/i)

\section{APOSTANDO POR LA REFLEXIÓN DECONSTRUCTIVA EN EL CAMPO EDUCATIVO}

Recogiendo los planteamientos de Foucault y Butler, en el artículo invitamos a la problematización de la norma sexual binaria contenida en la política de género y sexualidad en la educación chilena, en tanto dispositivo de control que refuerza la institucionalización/ normalización del dimorfismo sexual. Por ello, el uso de discursos de inclusión y de igualdad entre los sexos son analizados como reproductores de la separación entre las subjetividades dominantes y aquellas no contenidas en la categoría de normal (Matus e Infante, 2011). La dicotomía sexual en el discurso de las políticas educativas -la referencia reiterada a hombres y mujeres, así como a niños y niñas- sedimenta e institucionaliza -en términos de Berger y Luckmann (2008)- la segmentación sexual, perpetuando determinadas formas normativas de pensamiento legitimadoras de una determinada definición de la realidad. En la misma línea problematizadora del uso extendido en el lenguaje del binarismo sexual, Butler (2017) advierte del entrampamiento al que conduce la utilización por parte del feminismo de "las mujeres" como sujeto de éste. Como ella lo advierte, categorías de este tipo esencializan, creando la idea de una identidad común y de una universalidad entre quienes llevan dicha marca. Preguntarse ¿qué es ser mujer?, ¿qué es ser hombre? Son cuestiones que giran en las discusiones actuales dentro de los estudios de género, especialmente los queer. 
Como señalaAplablaza (2015) discursos comolos analizados en este artículo "perpetúan el statu quo(sic) de los grupos marginados, excluidos o subyugados, en su condición de ser 'otro' 'diferente' en espera a ser incluido por sujetos 'normales-privilegiados' (p. 263).

Desde la perspectiva posestructuralista se enfatiza en la construcción discursiva de la realidad y por ello, para desmantelar los procesos de constitución que han operado en el juego de su objetivación, se recurre a la historicidad a través de la deconstrución. El término 'deconstrucción' es la traducción que propone Derrida del término alemán Destruktion, que Martin Heidegger emplea en 1922 en el Informe Natorp y años después, en 1927, en su obra Ser y tiempo. Heidegger (2012) señalará que:

la hermenéutica cumple su tarea sólo a través de la destrucción (...) se ve obligada a asumir la tarea de deshacer el estado de la interpretación heredado y dominante, de poner de manifiesto los motivos ocultos, de destapar las tendencias y las vías de interpretación no siempre explicitadas y remontarse a las fuentes originarias que motivan toda explicación por medio de una estrategia de desmontaje (p. 51).

Heidegger alude a la destrucción del contenido tradicional de la ontología antigua, refiriéndose a "la investigación de la 'partida de nacimiento' del ser, en busca de las experiencias originales en que se ganaron las primeras determinaciones del ser" (2012, p. 33). Señalará que en la pregunta misma que interroga por el ser hay que "ver a través de su peculiar historia, ablandar la tradición endurecida y disolver las capas encubridoras producidas por ella" (2012, pp. 32-33). La propuesta deconstructiva entonces consistirá en escudriñar en la historia -realizar una arqueología, una genealogía- que permita el develamiento de aquello que hemos venido creyendo como lo natural, como lo obvio. Como se dijo antes "comprender qué es una institución pasa por comprender el proceso histórico por el cual se produjo" (Berger y Luckmann, 2008, p. 74). La deconstrucción es entonces una propuesta subversiva, porque nos muestra que la verdad que aceptamos como única, natural y obvia, no lo es. La imposición estructural de unas ontologías cuyos fundamentos no escapan ni pueden escapar de su propia lógica discursiva, y que son la raíz de todo orden social.

Si las políticas no son neutras, sino que están orientadas por ciertos valores y representan supuestos específicos, cabe preguntarse por qué y para qué el binarismo sexual, así como preguntarse qué valores y qué tipo de sociedad es la que se pretende sostener bajo esa premisa: ¿segmentar y de ese modo esencializar?, ¿sostener otra norma: la heterosexual?, ¿sostener la reproducción, para mantener el privilegio de determinados grupos?

Los documentos analizados sostienen una crítica a la correspondencia obligatoria del sexo con el género; sin embargo, no hacen lo mismo con la norma sexual binaria. En ese aspecto es posible develar una correspondencia del binarismo con la heterosexualidad y de ésta con la reproducción. Así "la metanarrativa del género y de la diferencia sexual no se ve amenazada por el desvío, sino que éste más bien la confirma" (Ramírez Pavelic y Contreras Salinas, 2016, p. 242).

De allí que los escenarios escolares resultan constreñidos y homogeneizantes para el estudiantado, donde "la diferencia y la visibilidad son reprimidas y subalternizadas en pos de desarrollar una educación normalizadora" (Ramírez Pavelic y Contreras Salinas, 
2016, p. 237). De esta manera, la pluralidad de manifestaciones/expresiones corporales, sexuales y del deseo son acalladas, si no apartadas y agredidas. Es por esto por lo que la visibilización es un acto político que permite dar cuenta de la existencia y entrar en la negociación de significados y valoraciones.

Proponemos -tomando las palabras de Matus e Infante (2011)- provocar en los espacios educativos 'prácticas más intelectuales', que problematicen las objetivaciones naturalizadas, que se exploren las maneras en cómo se razona la normalidad y la diferencia; cuestionando el para qué y para quiénes han servido y sirven; y que se promueva en el estudiantado y entre el profesorado indagar en cómo lo normativo ha sido histórica y culturalmente construido (Matus y Haye, 2015). En esa misma línea Apablaza (2015) invita a "cuestionar la normatividad aun presente en los contextos educativos, los discursos que circulan y aseguran el funcionamiento de tales modos de clasificación" (p. 264). Y por su parte Ramírez Pavelic y Contreras Salinas (2016) abogan por instalar en el campo educativo la necesidad de potenciar la reflexión y deconstrucción de la supuesta normalidad, así como eliminar los binarismos que "tanto parecen gustarle al constructo patriarcal, adulto/niño, hombre/mujer, heterosexual/homosexual” (p. 252).

La deconstrucción de los discursos del poder dominante no implica como resultado la desaparición de dichas narrativas. Sin embargo, la inclusión de las pluralidades posibilitará que sujetos diversos, cuenten con recursos, con medios para participar en la construcción de significados y nuevas definiciones de realidad. Hablamos de las condiciones para la circulación de discursividades -parciales y fragmentarias, no totalizadoras-, que den lugar a la expresión de constelaciones de socio-realidades antes invisibilizadas; las que, sin embargo, no quedan exentas de confrontarse en los juegos de poder y verdad.

\section{REFERENCIAS BIBLIOGRÁFICAS}

Ahmed, S. (2011). Problematic Proximities: Or Why Critiques of Gay Imperialism Matter. Feminist Legal Studies, 19(2), 119-132. DOI: 10.1007/s10691-011-9180-7

Ambrosy, I. (2012). Teoría Queer: ¿Cambio de paradigma, nuevas metodologías para la investigación social o promoción de niveles de vida más dignos? Revista Estudios Pedagógicos, 38(2), 277 285. http://dx.doi.org/10.4067/S0718-07052012000200017

Apablaza, M. (2015). El orden en la producción de conocimiento: Normatividades en la educación chilena en torno a diversidad. Revista Estudios Pedagógicos, XLI(Especial), 253-266. http:// dx.doi.org/10.4067/S0718-07052015000300016

Arriagada, I. (2006). Cambios de las políticas sociales: políticas de género y familia. Santiago de Chile: Naciones Unidas-CEPAL.

Berger, P. y Luckmann, Th. (2008). La construcción social de la realidad. Buenos Aires: Amorrortu.

Botero, A. M. A. y Duque, A. (2012). Construcción social de la identidad. Un acercamiento desde una perspectiva múltiple, Filo de la Palabra, (11), 27-36. Última visita 12 diciembre de mayo de 2018. Recuperado desde http://revistasum.umanizales.edu.co/ojs/index.php/filodepalabra/ article/viewFile/911/1036

Butler, J. (2017). El género en disputa. El feminismo y la subversión de la identidad. Buenos Aires: Paidós.

Foucault, M. (1985). Herculine Barbin llamada Alexina B. Madrid: Editorial Revolución. . (2000). Los anormales. Buenos Aires: Fondo de Cultura Económica. (2002). La arqueología del saber. Buenos Aires: Siglo Veintiuno Editores. . (2010). El orden del discurso. Barcelona: Fábula Editores. 
Fraser, N. (2008). La justicia social en la era de la política de identidad: redistribución, reconocimiento y participación. Revista de Trabajo, (6), 83-99. Última visita 8 de agosto de 2018. Recuperado desde http://trabajo.gob.ar/downloads/igualdad/08ago-dic_fraser.pdf

Galaz, C., Troncoso, L. y Morrison, R. (2016). Miradas críticas sobre la intervención educativa en diversidad sexual. Revista Latinoamericana de Educación Inclusiva, 10(2), 93-111. https:// dx.doi.org/10.4067/S0718-73782016000200007

Heidegger, M. (2012). El ser y el tiempo. Argentina: Fondo de Cultura Económica.

Infante, M., Matus, C. y Vizcarra, R. (2011). Razonando sobre la idea de diferencia en las políticas educativas chilenas, Universum, 26(2), 143-166. http://dx.doi.org/10.4067/S071823762011000200008

Iñiguez, L. (2005). Nuevos debates, nuevas ideas y nuevas prácticas en la psicología social de la era "post-construccionista". Athenea Digital, (8). Última visita 5 de mayo de 2017. Recuperado desde https://ddd.uab.cat/pub/athdig/15788946n8/15788946n8a15.pdf

Lamas, M. (2015). La antropología feminista y la categoría género. En M. Lamas (Comp), El género. La construcción cultural de la diferencia sexual, (pp. 93-122). México: Bonilla Artiga Editores y

UNAM, Programa Universitario de Estudios de Género.

Lyotard, J.F. (2000). La condición postmoderna. España: Cátedra.

Matus, C. (2018, julio 12) ¿Cómo y dónde ocurre la brecha de género? La Tercera. Última visita 10 de octubre de 2018. Recuperado desde https://www.latercera.com/opinion/noticia/dondeocurre-la-brecha-genero/240208/

Matus, C.y Haye, A. (2015). Normalidad y diferencia en la escuela: Diseño de un proyecto de investigación social desde el dilema político-epistemológico. Revista Estudios Pedagógicos, 41(Especial), 135-146. http://dx.doi.org/10.4067/S0718-07052015000300009

Matus, C. e Infante, M. (2011). Undoing Diversity: Knowledge and Neoliberal Discourses in Colleges of Education, Discourse, 32(3), 293-307. https://doi.org/10.1080/01596306.2011.573248

Ministerio de Educación, Gobierno de Chile. (2012). Enfoque de género en el espacio educativo. Santiago de Chile: MINEDUC. Última visita 28 de enero de 2017. Recuperado desde https:// bibliotecadigital.mineduc.cl/handle/20.500.12365/611?show=full

. (2013). Educación para la igualdad de género. Plan 2015-2018. Santiago de Chile: MINEDUC. Última visita 30 enero 2017. Recuperado desde https://www.mineduc.cl/wpcontent/uploads/sites/19/2017/01/CartillaUEG.pdf

(2015). Formación en sexualidad, afectividad y género. Santiago de Chile: MINEDUC. Última visita 28 de enero de 2017. Recuperado desde https://media.mineduc.cl/wp-content/ uploads/sites/28/2017/07/Formaci\%C3\%B3n-en-Sexualidad-Afectividad-y-G\%C3\%A9nero. pdf

(2017). Circular Derechos de niñas, niños y jóvenes trans en el ámbito de la educación. Santiago de Chile: MINEDUC. Última visita 30 de enero de 2017. Recuperado desde https:// www.supereduc.cl/wp-content/uploads/2017/04/ORD-N\%C2\%BA0768-DERECHOS-DENI\%C3\%91AS-NI\%C3\%91OS-Y-ESTUDIANTES-TRANS-EN-EL-\%C3\%81MBITO-DELA-EDUCACI\%C3\%93N-A-SOSTENEDORES.pdf

Martin Casares, A. (2018). Antropología del género. Cultura, mitos y estereotipos sexuales. España: Ediciones Cátedra, Universidad de Valencia.

Provoste, P. (2006). Equidad de género y reformas educativas: Argentina, Chile, Colombia, Perú. Santiago de Chile: Hexagrama.

Raíces Montero, J. H. (2015). Epistemología de las intersexualidades. En J.H. Raíces Montero (comp), Un cuerpo, mil sexos: intersexualidades (pp. 16-35). Ciudad Autónoma de Buenos Aires: Editorial Topías.

Ramírez Pavelic, M. y Contreras Salinas, S. (2016). Narrativas de identidad afectivo-sexual LGTB en contextos escolares: el aparecer frente al Otro. Revista Estudios Pedagógicos, 42(1), 235-254. http://dx.doi.org/10.4067/S0718-07052016000100015 
Rubin, G. (1986). El tráfico de mujeres: notas sobre la "economía política" del sexo. Revista Nueva Antropología, VIII(30), 95-145. Última visita 8 de mayo de 2017. Recuperado desde http://www. caladona.org/grups/uploads/2007/05/E1\%20trafico\%20de\%20mujeres2.pdf

Youdell, D. (2011). School Trouble. Identitity, power and politics in education. New York: Routledge.

Zúñiga, Y. (2017, julio 18). La ideología de género. El Mostrador. Última visita 28 de enero de 2018. Recuperado desde http://www.elmostrador.cl/noticias/opinion/2017/07/18/la-ideologiade-genero/ 
